\title{
Czochralski-Grown Silicon Crystals for Microelectronics
}

\author{
A. BuKOWsKi \\ Institute of Electronic Materials Technology, Wólczyńska 133, 01-919 Warsaw, Poland
}

\begin{abstract}
The Czochralski method of crystal growth is used since 1950s in scientific and industrial laboratories for growth of single crystals of large size and high quality. The article presents the general characteristics and selected improvements of the Czochralski method, and discusses its meaning and advantages in growth of silicon single crystals playing a key role in microelectronics.
\end{abstract}

DOI: $10.12693 /$ APhysPolA.124.235

PACS: 81.10.Fq, 85.40.-e

\section{The Czochralski method of silicon crystallization}

In the era of semiconductor electronics, monocrystalline silicon remains a basic and widely used material [1-4]. It will probably continue to dominate in the world of electronics for many years to come. The first material in history, though, on the basis of which the invention of a transistor was made (1948) was germanium (Ge). In order to produce a germanium single crystal, the molten germanium was held in a graphite crucible, and the crystal grew from a seed initially immersed in the liquid and later slowly pulled upwards. This method of crystallization was described in 1916 by Jan Czochralski, a scientist and inventor (for his life and scientific achievements, see [5]). During the first years after invention, Czochralski focused his studies on determination of the rate of metal crystallization. In later investigations, he proved that materials produced using this method are single crystals. They were not single crystals in today understanding, though, they were "threads" of such metals as zinc, tin and lead. At the time there was no need whatsoever for monocrystaline materials, as all materials industrially exploited at that time were polycrystalline. Properties were studied such as textures, martensitic structures; recrystallization, mostly in order to improve the mechanical properties.

Returning to the subject of this article, it must be pointed out that despite technological progress and development in the examination of semiconductor devices, the Czochralski crystallization method applied to semiconductor materials, in particular to silicon, remained essentially the same since the $1950 \mathrm{~s}$. It is commonly called the Czochralski method (for a detailed description of the method see, e.g. [6-8]). On the following pages, the characteristics of the method, contributing to its success are discussed, taking into consideration the mechanisms of crystallization and technological progress observed in the last decades. The discussion is focused on crystallization of silicon, a material which became irreplaceable in electronics and microelectronics. At the present time, silicon crystals are produced in tens of thousands of tons, annually. The broad use of silicon is due to both, its physical and technological characteristics as well as availability of resources (the primary resource of silicon is $\mathrm{SiO}_{2}$ ).

Silicon has a unique characteristics of forming, on its surface, an ultrathin passivating oxide film. Thermal conductivity of silicon $(140-163 \mathrm{~W} /(\mathrm{m} \mathrm{K}))$ is higher in comparison to other semiconductors (GaAs - 46$55 \mathrm{~W} /(\mathrm{m} \mathrm{K}), \mathrm{SiC}-16-55 \mathrm{~W} /(\mathrm{m} \mathrm{K}))$. This property is of particular significance as it leads to (advantageous) heat dissipation at semiconductor junctions. Silicon became a leading material in electronics due to its energy gap $(1.1 \mathrm{eV}$ at $300 \mathrm{~K})$ which guarantees the work of semiconductor devices at high temperatures.

\section{Growth process of silicon single crystals: basic parameters}

From the point of view of crystallization, the Czochralski method is classified as a directional crystallization process. During growth, the total mass of the material (liquid plus crystal) is conserved. If the crystal is doped, then the dopant concentration varies along its axis. The distribution of the dopant together with increasing solid:liquid proportion is described by the following formula [9]:

$$
C=k C_{0}(1-g)^{k-1},
$$

where $C$ - dopant concentration, $k$ - coefficient of dopant distribution, $C_{0}$ - concentration of the dopant before the beginning of the process, $g$ - the crystallized material to charge ratio.

General characteristics of commercially grown silicon single crystals includes the data on typical dopants, crystal orientation, growth rate and other technological parameters as well as on the quality and purity:

Technological parameters:

- Typical dopants: boron (B), phosphorus (P), antimony $(\mathrm{Sb})$ and arsenic (As),

- Crystal growth directions: $\langle 111\rangle,\langle 100\rangle,\langle 110\rangle$, $\langle 112\rangle$,

- Atmosphere: most often argon (Ar). Gas flow rate of $15-50 \mathrm{l} / \mathrm{min}$ most often in exhaust vacuum of 10-200 Tr.

- Rate of crystallization: $0.5-2.0 \mathrm{~mm} / \mathrm{min}$, 
- The charge and crystal diameter: up to $150 \mathrm{~kg}$ and $300 \mathrm{~mm}$, respectively,

- Homogeneous temperature distribution: achieved through both, the crucible rotation (usually 3-20 rpm) and rotation of pulling rod (with a Si seed attached) rotation (usually $5-30 \mathrm{rpm}$ ),

- Crucible material: usually pure "melted" quartz (rarely synthetic quartz),

- Material of heater and container for quartz crucible: high purity graphite,

- Liquid level during growth: typically, automated maintenance of a fixed level of the liquid is applied.

Characteristics of grown Si crystals:

- Typical structural quality: dislocation-free crystal.

- Usual carbon content: $<2 \times 10^{16}$ at. $/ \mathrm{cm}^{3}$,

- Usual oxygen content: $5 \times 10^{17}<\mathrm{O}_{2}<9 \times 10^{17}$ at. $/ \mathrm{cm}^{3}$.

\section{Silicon crystal growth process requirements. Characteristics of the Czochralski method}

Silicon (melting point $1415^{\circ} \mathrm{C}$ ) reacts with oxygen and water vapor if they are present, even in trace amounts, in the furnace atmosphere. It also enters into a live reaction with crucible materials.

In order to avoid oxidation of the charge, it is necessary to use a protective atmosphere, i.e. argon or helium with minimized oxygen and water vapor content. Molten silicon is an aggressive material, which dissolves the quartz crucible. The quartz crucible is a source of oxygen created due to the reaction of molten silicon with quartz. The dissolved oxygen partially leaves the liquid and moves to the furnace atmosphere, and forms the silicon monoxide. During the growth process, precipitate nucleation centers from foreign phases (i.e. silicon monoxide) affect the growth process. When such centers exist, the crystal continues to grow as a polycrystal. To ascertain a successful growth process, the formation of the nucleation centers must be minimized. A particular solution which reduces the nucleation centers formation is the direction of argon flow through the furnace. The gas is forced through the furnace against the convection stream, it is injected as if the smoke into a chimney and it is removed from the bottom part of the furnace using the suitable vacuum pumps. This is a particular solution the aim of which is to remove the nucleation centers (due to the presence of silicon vapor, silicon monoxide, and vapor of dopants such as phosphorus, antimony and other contaminations occurring at the crystallization front) from the area of crystallization. Facing the above defined difficulties requires application of various high-technologies such as: high vacuum technology, methods of pure argon production, methods of tightness assessment of installations and furnaces, electronic solutions of temperature stability processes, stability of movement parameters of the pulling rod, computerization of processes etc.

Taking into consideration the above requirements the fulfillment of which is not easy, the question remains: what are the advantages of the Czochralski method? There are only three methods of crystallization: the Czochralski method $(\mathrm{Cz})$, the floating zone (FZ) method and the Verneuil method (MV), which fulfill the condition that the growing crystal has no contact with the crucible material. Among these three methods of crystallization, two (Czochralski and floating zone) are useful for growth of silicon single crystals and ensure full repeatability of the growth process. The area of contact with the wall of the crucible is usually outlying from the area of crystallization and varies from a few to over ten centimeters. Such a large distance ensures a strong reduction of nucleation centers formation.

Another particular feature of the Czochralski method is the control of the diameter of the single crystal not only by changing the power of the heater, but also by changing the pulling rate or by changing the rotation speed of crucible or of the crystal. In the case of changing the pulling rate, the crystallization heat is emitted or absorbed, influencing the diameter of the growing crystal. This phenomenon does not occur when material crystallizes using a different method based on direct crystallization in a crucible. Appropriate manipulation or application of programming of the above-listed parameters not only changes the diameter of the growing crystal, but also activate or limit the nucleation centers.

\section{Improvements of the Czochralski method applied to silicon}

Modern microelectronics requires silicon single crystals of a standard $200 \mathrm{~mm}$ diameter (the state of the art technologies are starting to use the crystals of $300 \mathrm{~mm}$ diameter). The mass of $1 \mathrm{~m}$ long crystals exceeds $50 \mathrm{~kg}$. The quartz crucibles used in the growth process have a diameter of $18-24^{\prime \prime}(\approx 450-600 \mathrm{~mm})$. With increasing silicon charge, disadvantageous phenomena tend to occur, such as:

- Thermal turbulences appear in molten silicon of a large mass;

- Overheating of quartz crucibles is observed for crucibles of increased diameter and when the distance from the wall of the crucible to the side of growing single crystals is large;

- The large amount of molten silicon in itself has a negative influence on the crystal puller;

- Large electric power is needed to maintain a large mass of silicon in molten state. 
These factors entail large costs and cause difficulties in maintaining the high quality of single crystals. There are a few directions of improvement of the growth process of large crystals.

The first one is a group of improvements without changing the construction of the puller, for example using more than one heater, ameliorating the quartz crucibles, using new types of thermal insulation, constructional improvements of heating elements, etc.

Other improvements are more complex. Two of them are described below:

- Magnetic-field-applied Czochralski (MCZ), leading to significant reduction of the scale of turbulences, and consequently causing an improvement of single-crystal quality.

- Continuous liquid feed method (CLF method), resulting in an improvement of axial homogeneity of the single crystal. The method enables to reduce the mass of molten silicon.

\subsection{The Czochralski method with the application of a magnetic field}

The use of a magnetic field in silicon single crystal growth has a long history [10-12]. Research in this area and the first results of experiments were attained for single crystals of $76-100 \mathrm{~mm}$ diameter. The main aim, a reduction of the amount of oxygen in the silicon single crystals, has been achieved. For a long time, a steady magnetic field set horizontally (perpendicularly to the crucible axis) to the charge was used. In order to achieve the intended results, the intensity of the magnetic field was relatively large and amounted to $0.6-0.8 \mathrm{~T}$ which required an additional electric power comparable to the heating power of the furnace, despite using moderate-size chambers of Czochralski furnace.

The method was further developed using different configurations of magnetic field, i.e.:

(a) Horizontal magnetic field (mentioned above);

(b) Axial magnetic field (magnetic flux is parallel to the axis of the furnace and of the growing crystal);

(c) Unipolar magnetic field (the lines of the magnetic field are perpendicular to the walls and to the bottom of the quartz crucible). This effect was attained thanks to the interaction of two independent axial fields;

(d) Magneto-hydro-dynamic-rotor (MHD);

(e) Steady (axial) magnetic field with a simultaneous interaction of MHD configuration. The aim is to break down "the cells of flow circulation" which are formed in the axial field using the MHD configuration.
Due to the use of two configurations simultaneously, the magnetic fields described in (c) and (e), are called second-generation magnetic fields.

Up to now, the studies of magnetic field application have been mostly focused on the configurations (a), (b) and (c). Their characteristics can be summarized as follows.

\subsubsection{Steady magnetic fields - horizontal (a)}

When applying a relatively strong magnetic field such as $0.6-0.8 \mathrm{~T}$, a significant decrease of oxygen content in the crystal is attained. If such strong magnetic field is used, the processes are difficult to carry out because of problems related to the flow of heat from the heater to the crystallization area. The costs related to the application of such magnetic field are high. When a weaker magnetic field is applied (up to $0.05 \mathrm{~T}$ ) thermal convection was absent due to the rotation of the crucible and the seed crystal which is a significant advantage of this configuration [13].

\subsubsection{Axial magnetic field (b)}

The temperature layers under the crystallization front are more symmetrical than in configuration (a). Due to this, the growth processes are easier to carry out. The homogeneity and oxygen reduction effects are difficult to achieve. Slowing down the oxygen diffusion to the surface leads to an increased oxygen content in the grown crystals. Application of this configuration does not bring any advantages.

\subsubsection{Unipolar magnetic field (c)}

The lines of the magnetic field are orthogonal in relation to the bottom of the crucible and to its side. Due to this, the largest magnetohydrodynamic pressure occurs at the crucible walls, whereas small magnetic forces act on the area around the crystallization front. The drop of oxygen value to the level of $2 \times 10^{17}$ at. $/ \mathrm{cm}^{3}$ was achieved with magnetic field of $0.1 \mathrm{~T}$, which is the best result so far.

Besides the above mentioned effects, application of the magnetic field leads to a visible calming of the liquid surface. When the charge is large, vibrations of the extended liquid surface occur as a harmful phenomenon. Elimination of the vibrations is an additional advantage of magnetic field application.

\subsection{Continuous liquid feed method}

Research regarding this method originates from the needs to achieve savings in the use of the quartz crucible $[12,14]$. Continuous feeding of the crucible with silicon leads to crystals of a mass larger than the mass of original charge. Theoretically, when using the continuous feeding, the crystal length is limited only by the time during which the quartz crucible remains intact. In practice, several silicon single crystals of total $100-200 \mathrm{~kg}$ mass are grown in a series of processes proceeded without cooling the furnace. The furnace may be charged with polycrystalline rods, granules or silicon powder as well as with molten silicon. 
A practical problem that arose, regarded the process of a continuous feeding of melted silicon which caused perturbations in the process of crystal growth. So far, this method is used to produce silicon for solar applications, where the requirements concerning crystal quality are not high. If the perturbation problem was solved, this method would become of interest for the single-crystal growth task. Furthermore, besides savings in the use of the quartz crucible, this method opens the door to achieve a high homogeneity in axial distribution of the dopant. The silicon feed which does not contain a dopant, will dissolve the concentrated dopant in the liquid found in the crucible. In the case of dopants with a decomposition coefficient $k<1$, a constant concentration of, e.g., phosphorus in silicon crystal may be expected. This phenomenon had been confirmed by experimental data.

Crystallization at the conditions of strongly reduced volume of molten silicon in the crucible may become an even more valuable modification of the method. Here, the crucible will be fed with a "silicon feed". In this case, the volume of the molten material will be a classical "zone" and the crystallization will be subject to zone crystallization instead of conventional Czochralski directional crystallization. Consequently, the dopant distribution will be determined by a different law. Up to now, such a version of the CLF method has not been reported, but the prerequisites of such a method seem to be justified. The advantage of this version would be to achieve five times smaller volume of liquid in the crystallization area than while using the conventional method.

Such or other improvements are expected to be developed in future. They can be concentrated around new crucible materials (i.e. silicon nitride, carbon based materials, other materials and their combinations, including modified quartz). Using such new materials may be fruitful for the success of the CLF method.

\section{Summary}

In the article, the currently applied Czochralski technique of silicon single crystal production is presented. Multiple advantages of the method led to production of such crystals at an industrial scale. The increase of diameter of produced single crystals from $76-100 \mathrm{~mm}$ to 200-300 mm has been accompanied by various improvements, such as magnetic field application and continuous liquid feeding. It is expected that such improvements will be introduced into commercial production practice. In particular, the introduction of magnetic fields is ex- pected to take place soon, as multiple possible magnetic-field settings (especially the second generation fields) give a big choice of configurations. Moreover, for magnetic field generations, superconducting magnets at liquid helium temperature can be used with a reduced power cost. Good quality of silicon crystals produced using the MCZ method prove that its application is effective. In the CLF method the main efforts regarding its implementations and improvements are focused on the use of new materials, including, in particular, new crucible materials.

\section{References}

[1] Handbook of Semiconductor Silicon Technology, Eds. W.C.O. Mara, R.B. Herring, L.P. Hunt, Noyes Publications, Park Ridge (NJ) 1990.

[2] H.R. Huff, J. Electrochem. Soc. 149, S35 (2002).

[3] L. Arnberg, M. Di Sabatino, E. Øvrelid, JOM, 38 (October 2011).

[4] G. Fisher, M.R. Seacrist, R.W. Standley, Proc. IEEE 100, 1454 (2012).

[5] P. Tomaszewski, On Jan Czochralski, Atut, Wrocław 2012 (in Polish, the English translation in preparation).

[6] Elementary Crystal Growth, Ed. K. Sangwal, Saan Publ., Lublin 1994.

[7] D.T.J. Hurle, B. Cockayne, in: Handbook of Crystal Growth, Vol. 2A, Basic Techniques, Prentice-Hall, Englewood Cliffs 1994, Ch. 3.

[8] A.A. Wheeler, in: Handbook of Crystal Growth, Vol 1b. ed. D.T.J. Hurle, North-Holland, Amsterdam 1993, p. 683.

[9] W.G. Pfann, Zone Melting, Wiley, New York 1958.

[10] M. Ohwa, T. Higuchi, E. Toji, M. Watanabe, K. Homma, S. Takasu, in: Semiconductor Silicon, Eds. H.R. Huff, T. Abe, B. Kolbesen, The Electrochemical Society, Pennington, NJ 1986, p. 117.

[11] L.N. Hjellming, J.S. Walker, J. Fluid Mech. 182, 335 (1987).

[12] A. Bukowski, P. Zabierowski, Elektronika 40, 10 (1999) (in Polish).

[13] A. Bukowski, P. Zabierowski, W. Grzejszczyk, R. Nowak, Method of Obtaining the Silicon Single Crystals in the Czochralski Crystalization Device, Patent A1(21) 288359 (22) $9012 \quad 20 \quad 5(51) \quad$ C33B 15/00, 13.01.1994 (Polish patent).

[14] Leybold System Presentation - Silicon'96, Fifth Scientific and Bussiness Conf., Rožnov pod Radhoštěm (Czech Republic) 1996. 